Research Article

\title{
Estimation and Control for Industrial Products Reliability under Failure Trials
}

\author{
Kui Wang (iD) and Lili Ding $\mathbb{D i D}^{2}$ \\ ${ }^{1}$ School of Mechanical Science and Engineering, Huazhong University of Science and Technology, Wuhan 430074, China \\ ${ }^{2}$ Yichang Meteorological Bureau, Yichang 443000, China \\ Correspondence should be addressed to Lili Ding; dinglili1986@126.com
}

Received 29 September 2020; Revised 28 October 2020; Accepted 17 November 2020; Published 8 December 2020

Academic Editor: Chaoqun Duan

Copyright (c) 2020 Kui Wang and Lili Ding. This is an open access article distributed under the Creative Commons Attribution License, which permits unrestricted use, distribution, and reproduction in any medium, provided the original work is properly cited.

\begin{abstract}
In practices, most industrial products are subject to sudden failure and only failure information can be collected, which presents a great challenge for reliability prediction of modern devices. To address this issue, our paper proposes a dynamic reliability estimation and control for industrial products under regular failure trials. The failure trial is performed at different operational time points of the products, which provides sole data source for evaluating the status of industrial products. We use Bayesian approach to dynamically estimate the industrial products when the failure trial is available. The estimated reliability is updated using a point estimate with new available data. To maintain the reliability of products at a desirable status, a reliability control method is presented to monitor the confidence interval of reliability distribution. The lower limit of confidence interval is maintained above a control limit, which indicates that a corresponding quality-assurance action is preferable. The proposed reliability estimation and control approach is demonstrated using a case of light-emitting diodes under failure trials at production process. The obtained results indicate the effectiveness of our estimation and control model.
\end{abstract}

\section{Introduction}

With development of industrial engineering, small factories are becoming large-scale plants, which prompts the applications of prognostics [1]. For prognostics of the actual status of industrial products, reliability estimation is a crucial step, which provides risks information for the maintenance engineers $[2,3]$. The reliability of industrial products is the ability to complete their specified functions under specified time [4]. This index is also the prerequisite of implementation of the condition-based maintenance. To eliminate the failure occurrences of the industrial products, the reliability estimation is required to ensure the production quality in most industries [5].

Most reliability estimation works were mainly focused on the prediction stage; however, there was no practical or useful guidance for the real applications $[2,6]$. When we obtained estimated reliability level of industrial products, we rarely have useful policy to improve the reliability during the production process, where the reliability control approach is very much required in quality control process [7]. Therefore, this paper aims to propose a reliability estimation and control approach to address this problem existing in modern industries.

The consciousness of reliability applications has been noticeably improved in the recent years [8-10]. The reliability evaluation system can ensure many critical and heavy-duty machines with a relatively safe operating conditions, and it has become an indispensable part of modern industry [11]. This system is also widely used in manufacturing engineering, aviation, and nuclear industries $[11,12]$. To properly develop an effective reliability evaluation system for industrial products, the reliability prediction and control techniques should be explored and studied. Currently, there are some approaches related to reliability evaluation, which are the statistics approaches for reliability modeling including reliability block diagrams, degradation-based reliability 
estimation, and hazard-based analysis [13-15]. These existing works often modeled degradation path or hazard pattern as fixed model and used some ideal assumptions to compute the reliability of industrial products. Besides, the distribution of reliability is not provided in these mentioned research works. In a classical reliability framework, it is often difficult or impossible to apply single-unit estimation model for a complex system consisting of multiple units [16]. The reliability distribution and mean reliability are completely different for these two systems. This hinders the application of current estimation methods on complex systems. Furthermore, the parameters evaluated are unknown constants and they are evaluated only based on large amount of recorded data sample [17]. In some engineering practices, however, there could be no perfect sources of reliability data. For example, testing of rare and valuable products such as missiles and satellite systems, the sample size will be small or even missing [18]. To achieve an accurate reliability estimation from these data sources through classical statistics approaches can be a great challenge.

To overcome the above challenge, researchers have employed Bayesian statistical techniques for reliability estimation. The Bayesian statistical techniques use an updating process to renew the operating reliability of industrial products, and when the new observation data are collected, more accurate estimation is provided. The Bayesian reliability estimation technique is especially useful for system with small size of data sample [11]. Cole [19] proposed a reliability assessment method for industrial systems. In Cole's work, Bayes' theorem is used to determine posterior distribution of system reliability and update system condition level. Mastran et al. [20] proposed a reliability assessment method for coherent structure of industrial products. Failure data from structure, components, and system are used for updating the instant reliability. Sharma and Bhutani [21] studied the Bayesian reliability analysis of the parallel industrial system, and the prior information of the failure rate of the products is used for reliability assessment. Bao et al. [22] used the processing reliability data to analyze the reliability of high flux engineering test reactor. The data obtained from similar research reactors are selected as the Bayesian prior distribution to compute the Bayesian posterior distribution of the reliability. Gardner et al. [23] presented a Bayesian history matching technique in a structural dynamics context to infer the model discrepancy. The Gaussian process regression is utilized to map the simulator output and for training observation data. For summarizing the existing Bayesian statistical techniques, Insua et al. [11] have reviewed the existing Bayesian approach in reliability decision-making. They illustrated how the Bayesian approach is applied in life testing, experimental design, reliability certification, and preventive maintenance areas.

To achieve more accurate prediction, Peng et al. [24] proposed a generalized multivariate hybrid degradation model to incorporate and analyze the dynamic degradation. A two-step Bayesian framework is presented for parameter estimation. Moreover, Mahadevan et al. [25] presented a method of artificial intelligence called Bayesian network, which has been an effective tool for modeling uncertainty in reliability analysis and is commonly used in reliability analysis due to its powerful model structure [26]. Boudali et al. [27] have studied an improved reliability analysis method based on Bayesian network to model the dynamic degradation process of industrial systems. In this research, a discrete-time Bayesian network framework has been defined and proved to be a powerful tool for modeling and analyzing the behavior and interaction of various system components. Lee and Pan [28] employed the nonparametric Bayesian network, which is suitable to model any type of continuous and discrete random variables and overcomes the limitation provided by the discrete Bayesian network. Also, a Bayesian method combining the prior knowledge with expert opinions was proposed to evaluate the system reliability at the early design stage and is proved to be effective in a real case study. As an extension, Bayesian network has also been applied in the mechanical product quality improvement [29]. A Bayesian principle-empirical model fusing the principal knowledge and empirical data is presented to discover the relations of quality characteristics. Later, Cai et al. [30] have concluded the Bayesian network applications in reliability evaluation. This work mainly focused on the reliability modeling procedures with Bayesian network and provided guide for practitioners in practical implementations. More recently, Rebello et al. [31] combined a hidden Markov process and dynamic Bayesian network to present a hybrid reliability prediction method to overcome the difficulty in discretization and multiple variables representation. In this method, process data can be used in continuous time domain without discretization. These Bayesian approaches used in reliability engineering are verified to be an effective tool to use paucity of data in reliability prediction under ideal assumptions.

To fully use limited testing data in the production process, in this paper, we use a Bayesian procedure to dynamically estimate the reliability of the industrial products and present a reliability control method to maintain the lower limit of prediction interval above a certain level. This is the first paper to provide reliability estimation and control approach using Bayesian procedures. The main contributions of this work are as follows: (1) Interpretation of the past and present failure trials using inconstant general distribution, (2) development of a dynamic estimation procedure for updating products reliability, (3) development of a novel reliability control scheme based on confidence interval, and (4) validation of the improved performance using a real case study.

The remaining parts of this paper are organized as follows. In Section 2, industrial products failure trial is described. In Section 3, a reliability estimation model using Bayesian approach is presented for industrial products. In Section 4, a reliability control method is developed to maintain the reliability level of the products. In Section 5, a case of light-emitting diodes under failure trials at production process is illustrated to show the effectiveness of the proposed model. Finally, conclusions and future work directions are provided in the last section. 


\section{Industrial Products Failure Trials}

The industrial products are subject to regular failure inspection throughout the production process. The failure inspection is performed at discrete time points to reveal whether the examined product can be functional. The observed failure rate at different time point is a crucial index for quality assurance in production department of industry. This is a common practice for most modern industrial manufacturers, where the failure trials at regular time epochs provide important information for the reliability estimation and control of the industrial products. Figure 1 shows the flowchart of the failure trial in industry. The new manufactured products are inspected at different time with trial 1 to trial $n$ to examine the qualification rate of the product. The aim of the trial is to know the quality of the new products at each service time.

The failure trial samples a constant number of products and examines the quality of the products at each trial. Through the failure trial, the number of qualified products is obtained, which provides the information for maintenance engineers to estimate the reliability of the batch of products. Figure 2 shows the results of failure trials at each trial epoch. It can be observed that the qualified products at each trial present a shock tend, which signifies that the quality of new products is not stable and strict control should be applied.

We assume that, for a given trial, the overall number of tested products is $S$, and the qualified number of the tested products is $q$. For that specified configuration, the probability that such a test result is obtained is conditioned on previous configuration $x$ :

$$
P(S, q \mid X=x)=x^{q} \cdot(1-x)^{S-q},
$$

where $X$ denotes the probability that a product is successful on a given trial.

To make full use of the failure trials data, we consider the Bayesian approach to use the discrete trial information to dynamically update the reliability of the tested products, and the procedure is presented in the next section.

\section{Reliability Estimation}

For a given trial, the Bayesian approach can infer an approximate value of the product reliability. As the trial continues, the value is renewed, and more accurate reliability estimation can be obtained. For Bayesian approach, the typical Bayes' theorem can be expressed as

$$
P\left(A_{i} \mid B\right)=\frac{P\left(B \mid A_{i}\right) P\left(A_{i}\right)}{\sum_{\text {all } j} P\left(B \mid A_{j}\right) P\left(A_{j}\right)},
$$

where $P$ indicates the probability of , and $B$ is any trial with positive probability. For the case where $X$ and $Y$ are both continuous random variables, equation (2) can be equivalent to

$$
f_{X \mid Y}(x \mid y)=\frac{f_{Y \mid X}(y \mid x) f_{X}(x)}{\int_{-\infty}^{\infty} f_{Y \mid X}(y \mid x) f_{X}(x) \mathrm{d} x},
$$

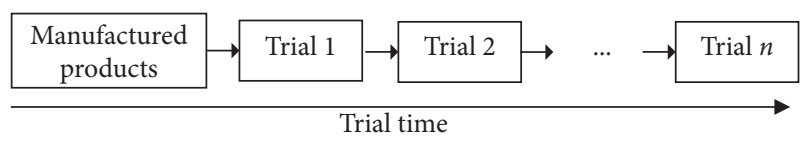

FIGURE 1: Flowchart of failure trial process in industry.

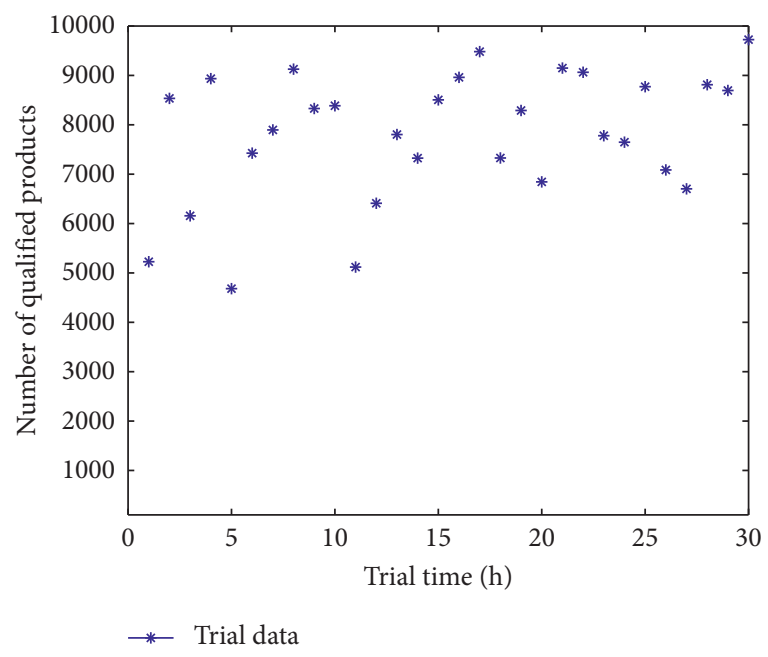

FIGURE 2: Failure trial of the industrial products.

where $f_{X}$ represents the probability density function of the random variable $X$ and $f_{X \mid Y}$ is a conditional probability density function, which is conditioned by a value of the random variable $Y$.

If $X$ is continuous and $Y$ is discrete, equation (2) can be written as

$$
f_{X \mid Y}(x \mid y)=\frac{P(Y=y \mid X=x) f_{X}(x)}{\int_{-\infty}^{\infty} P(Y=y \mid X=x) f_{X}(x) \mathrm{d} x} .
$$

For the failure trial of industrial products, $f_{X}(x)$ in equation (4) represents the density of the random variable of $X$ before the next trial data are collected, and it is called the a priori density of $X . P(Y=y \mid X=x)$ is the probability of specified trial data given that the random variable has the value $x . f_{X \mid Y}$ is the a posteriori probability density of $X$ given $Y$.

In the application of Bayes' theorem to reliability, the probability that, for a given trial, a product is qualified for manufacturing is denoted by $R . R$ is treated as a random variable, since the product reliability is not constant and will be decreased with service time.

We suppose that the product reliability is a continuous random variable between 0 and 1 . For overall $S$ tested products, the reliability of the batch of product can be inferred as

$$
f_{R \mid r_{S}}(r \mid q ; S)=\frac{r^{q}(1-r)^{S-q} f_{R}(r)}{\int_{0}^{1} \beta^{q}(1-\beta)^{S-q} f_{R}(\beta) \mathrm{d} \beta},
$$

where $r$ for $0 \leq r \leq 1$ represents the value of estimated reliability. $r_{S}$ represents the qualified number of products in all $S$ tested products. $f_{R}$ is the a priori density of the random variable $R$. Recalling equation (1), to compute with the 
Bayesian approach, $f_{R}(r)$ can be considered as a random distribution with general form, such as Beta distribution and uniform distribution. Since the support interval of Beta distribution lies in $[0,1]$, it should be a natural candidate to model $f_{R}(r)$. Then $f_{R}(r)$ can be of the following form:

$$
f_{R}(r)=\frac{r^{k}(1-r)^{l}}{\int_{0}^{1} \beta^{k}(1-\beta)^{l} \mathrm{~d} \beta}
$$

where $k$ and $l$ are the positive integers. The denominator of the above equation can be written in the following form:

$$
\begin{aligned}
\int_{0}^{1} \beta^{k}(1-\beta)^{l} \mathrm{~d} \beta & =\frac{\Gamma(k+l+2)}{\Gamma(k+1) \Gamma(l+1)} \\
& =B(k+1, l+1), \\
& =\frac{k ! l !}{(k+l+1) !}
\end{aligned}
$$

Therefore, the reliability conditioned on the available trial data can be derived using Bayes' theorem, which is given by

$$
f_{R \mid r_{S}}(r \mid q ; S)=\left(\frac{(S+k+l+1) !}{(q+k)(S+l-q)}\right) r^{q+k}(1-r)^{S-q+l} .
$$

The reliability of the industrial products is obtained using equation (8), which indicates the estimated reliability distribution. To make the Bayesian updates less complicated, we use point estimates to represent the renewed mean reliability level of each trial. The point estimates use the expected value of the distribution of Bayesian a posteriori probability. In the following, we present a point estimation approach to simplify the Bayesian updating process.

The representative of the Bayesian reliability is considered here, and using differential calculus, equation (8) has the point estimate given by

$$
\frac{k+q}{k+l+S}
$$

The above expression represents the updated mean reliability level at each sampling time epoch. When the a priori density is chosen as uniform, that is, $k=l=0$, the estimate is simply $(q / S)$. The uniform a priori distribution is an ideal situation that may not happen in reality. Thus, a second point estimate with more practical characteristics is presented, which is called the Neyman estimate [32]. The mean of the a posteriori density derived from equation (8) is given by

$$
\frac{k+q+1}{k+l+S+2}
$$

In this instance, equation (10) is commonly referred to as the Bayesian estimate of the product reliability. We use equation (10) to calculate the reliability of industrial products at each failure trial.

\section{Reliability Control}

When we have obtained the expression of Bayesian estimate for the product reliability, we aim to maintain the satisfied level of product reliability. To maintain the reliability of product above a certain level, we propose a method to find optimal range of reliability distribution. The prediction interval of reliability distribution indicates the cumulative survival probability of the products. The method controls the cumulative survival probability at each failure trial to ensure that the risk of hazard is below a certain level $\alpha$. Since the upper limit of range of reliability distribution is 1 , we need to monitor the lower limit of the reliability distribution range, which ensures the cumulative survival probability above $1-\alpha$. When the lower limit of reliability distribution falls out of the range, the method alarms to indicate that a corresponding quality-assurance action is required. Using a Bayes technique, the two-sided $100(1-\alpha)$-precent confidence limits on $R$ are defined as

$$
P\left(R_{\text {Lower }} \leq R \leq R_{\text {Upper }}\right)=\int_{R_{\text {Lower }}}^{R_{\text {Upper }}} f_{R \mid r_{S}}(r \mid q ; S) \mathrm{d} r=1-\alpha .
$$

subject to $R_{\text {Upper }}-R_{\text {Lower }}$ being a minimum, where $R_{\text {Upper }}$ denotes the upper limit of estimate distribution of reliability and $R_{\text {Lower }}$ denotes the lower limit of the distribution.

In reliability engineering, the high-quality product requires that the upper limit of reliability be equal to one, which means that the probability of survival product at certain time might be one; that is, $R_{\mathrm{Upper}}=1$. The lower onesided limit is given by

$$
P\left(R_{\text {Lower }} \leq R\right)=\int_{R_{\text {Lower }}}^{1} f_{R \mid r_{S}}(r \mid q ; S) \mathrm{d} r=1-\alpha .
$$

Referring to equation (8), we consider the a priori density of $R$ as a uniform $[0,1]$; that is, $k=l=0$. Equation (12) can be derived as

$$
P\left(R_{\text {Lower }} \leq R\right)=\int_{R_{\text {Lower }}}^{1} \frac{r^{q}(1-r)^{S-q}}{B(q+1, S-q+1)} \mathrm{d} r=1-\alpha .
$$

The integral in equation (13) is the incomplete Beta function $I_{R_{\text {Lower }}}(q+1, S-q+1)$, and the values of incomplete Beta function are presented in [33]. For any $I_{R}(q+1, S-q+1)$, the expression can be further extended as

$$
I_{R}(q+1, S-q+1)=\sum_{x=q+1}^{S+1}\left(\begin{array}{c}
S+1 \\
x
\end{array}\right) R^{X}(1-R)^{S-X+1} .
$$

Therefore, for the $100 \alpha$-percent lower limit for $R, R_{\text {Lower }}$ is given by the root of the following expression:

$$
\sum_{x=q+1}^{S+1}\left(\begin{array}{c}
S+1 \\
x
\end{array}\right) R^{X}(1-R)^{S-X+1}=\alpha .
$$


At this point, we can use the existing table for incomplete Beta function to give limits on $1-R$ rather than $R$. Using a similar method, the expression for the $100 \alpha$-percent upper limit on $U=1-R$ can be obtained by the root of

$$
\sum_{y=S-q+1}^{S+1}\left(\begin{array}{c}
S+1 \\
y
\end{array}\right) U^{y}(1-U)^{S-y+1}=1-\alpha,
$$

where $y=S-q+1$. To further derive the confidence limit of $U$, we use Neyman interval [29], and the $100 \alpha$-percent upper limit of confidence can be computed using the root of the following equation:

$$
\sum_{y=S-q+1}^{S}\left(\begin{array}{l}
S \\
y
\end{array}\right) U^{y}(1-U)^{S-y}=1-\alpha .
$$

Contrary to equation (16), it is shown that the Neyman interval with $S-q$ failures (unqualified products) in $S+1$ trials is the same as Bayes estimate interval with assumptions of a uniform a priori density on $R$ under $S-q$ failures in $S$ trials. In the case that the a priori density of $R$ is chosen as a Beta density, we have

$$
f_{R}(r)=\frac{r^{k}(1-r)^{l}}{B(k+1, l+1)} .
$$

For the lower $100 \alpha$-percent confidence limit on $R, R_{\text {Lower }}$ is defined as

$$
\begin{gathered}
\int_{R_{\text {Lower }}}^{1} \frac{r^{q+k}(1-r)^{S-q+l}}{B(q+k+1, S-q+l+1)} \mathrm{d} r=1-\alpha, \\
1-I_{R_{\text {Lower }}}(q+k+1, S-q+l+1)=1-\alpha .
\end{gathered}
$$

It is very clear that, with simple computation, the $100 \alpha$-percent upper limit on $U=1-R$ can be determined by the solution of the following equation:

$$
\sum_{y=S-q+l+1}^{S+k+l+1}\left(\begin{array}{c}
S+k+l+1 \\
y
\end{array}\right) U^{y}(1-U)^{S-y+k+l+1}=1-\alpha .
$$

Now we have obtained the range of confidence interval. To maintain the reliability level of the industrial products, we set $\bar{R}$ as control limit of the reliability. When the lower limit of reliability distribution decreases to be below $R_{\text {Lower }}$, that is, $\bar{R}<R_{\text {Lower }}$, a corresponding quality assurance is required. The determination of $\bar{R}$ can be achieved using an algorithm presented in [34].

The confidence interval of reliability on a given operational time is updated at every trial, and the lower limit of the confidence interval should be maintained above a certain level $\bar{R}$, which guarantees the quality of industrial production process. In the next section, we use a case to illustrate how to estimate the product reliability and control the reliability level in the production process.

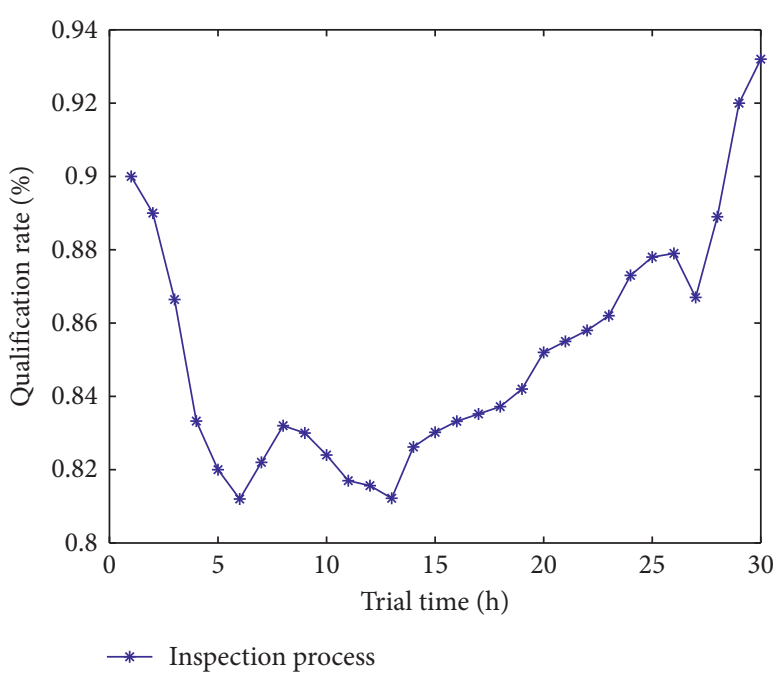

FIgURE 3: Qualification rate of each trial.

\section{Case Study}

5.1. Case Preprocessing. To illustrate the proposed approach, a case of light-emitting diodes was chosen to test the performance of our model. The failure trial (i.e., qualification test) was performed every 20 hours after the light-emitting diodes were produced. The failure trial is to ensure that the quality of the light-emitting diodes is maintained at a desired level. When the reliability of the light-emitting diodes derived from failure trial drops to a danger level, quality assurance action is initiated to check and guarantee the qualification rate of the production process. In each trial, the quality engineer took a sample size of products, and the sample size was not fixed for each trial. The light-emitting diodes were tested with result of "qualify" or "unqualify/ failure." The qualification rate (i.e., $(q / S)$ ) of each trial is computed and indicated in Figure 3. In Figure 3, the failure trial is performed 30 times, up to 600 testing hours. We use the data presented in Figure 3 to estimate the product reliability in each trial.

We consider the initial value of Bayesian estimate as the qualification rate of 1st failure trial, which is set to 0.902 . The upper limit of reliability prediction range is considered as 1 . The reliability of light-emitting diodes at each trial is updated using (9) in Section 3.

5.2. Reliability Prediction. Using the approach presented in Section 3, the reliability of light-emitting diodes can be obtained by equations (8)-(9). The estimated reliabilities at each trial are illustrated in Figure 4. In the first row of Figure 4, the mean reliability of the light-emitting diodes at the 5th trial is 0.816 , which has been decreasing since the 1st trial. At the 10th trial, the distribution of reliability range is wider and scatter, which means that the quality of light-emitting diodes is not stable at this stage. In the second row of Figure 4, the reliability of light-emitting diodes has been slightly improved and increased to 0.9 at 


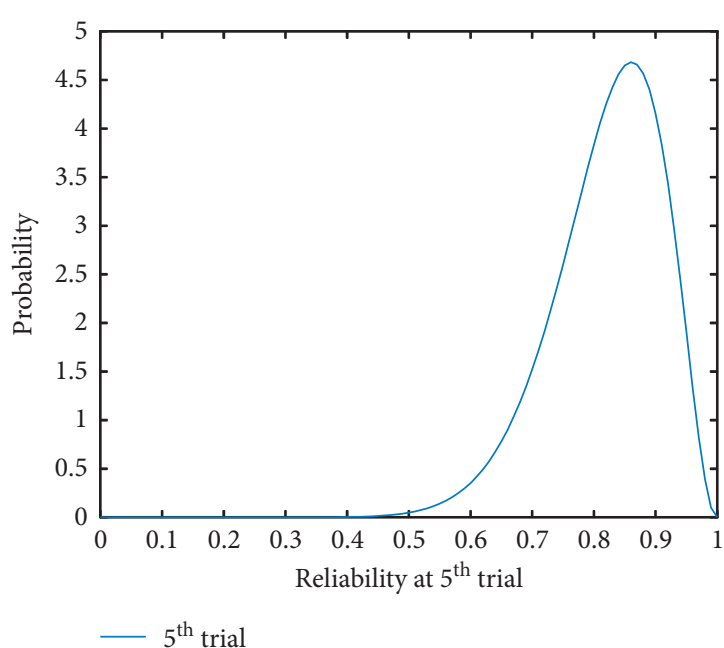

(a)

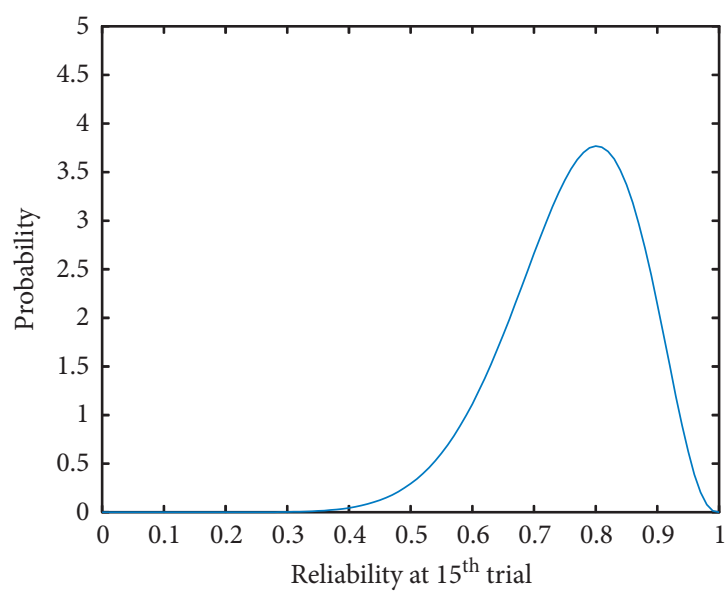

$-15^{\text {th }}$ trial

(c)

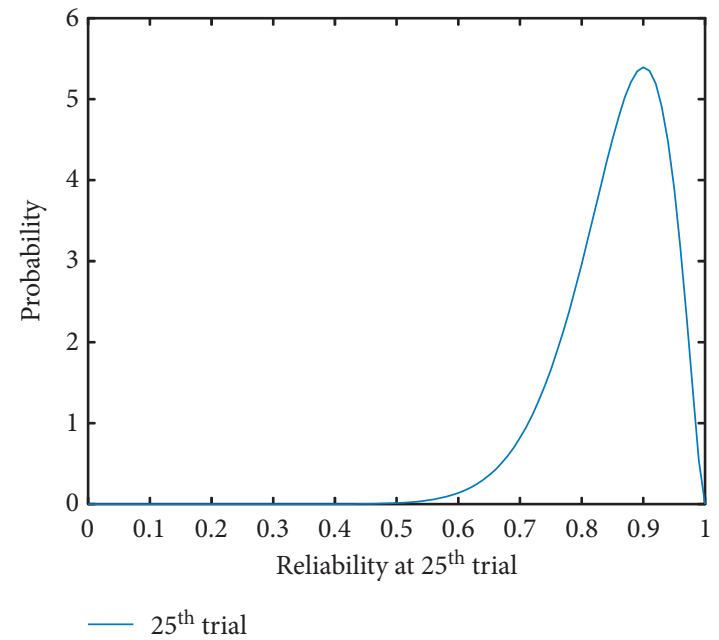

(e)

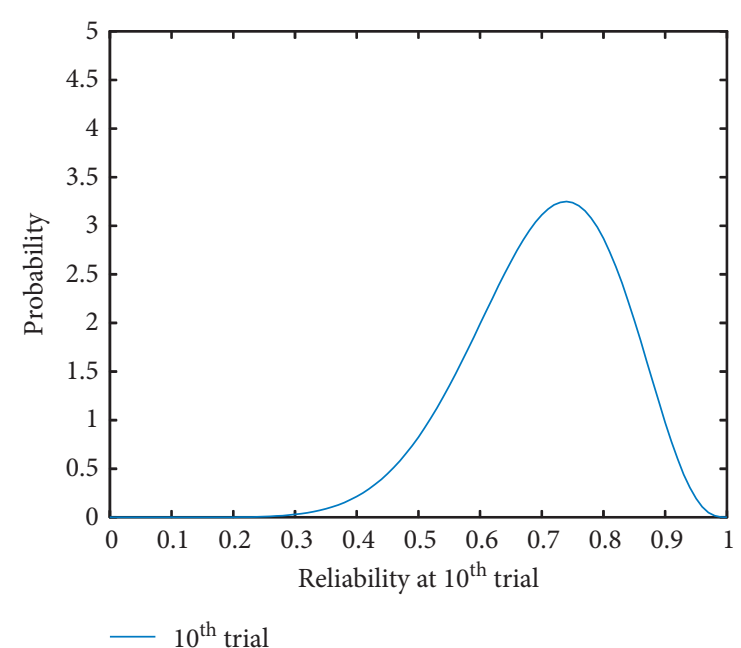

(b)

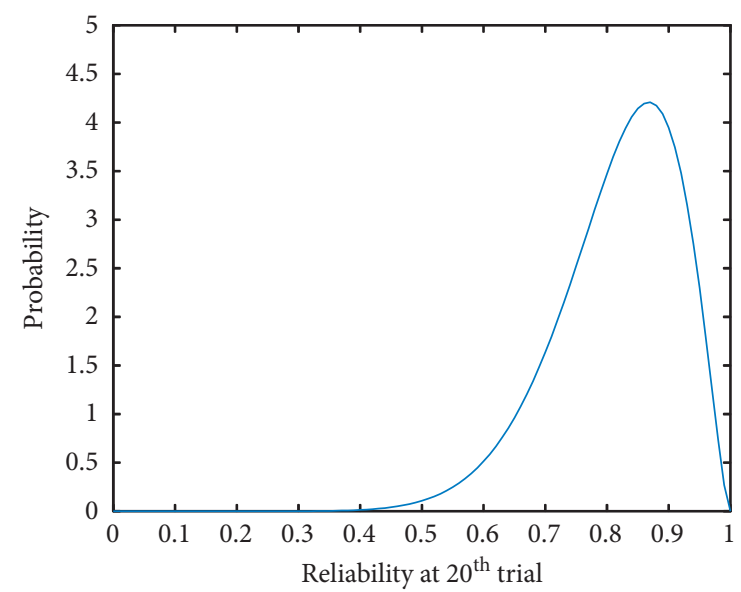

$-20^{\text {th }}$ trial

(d)

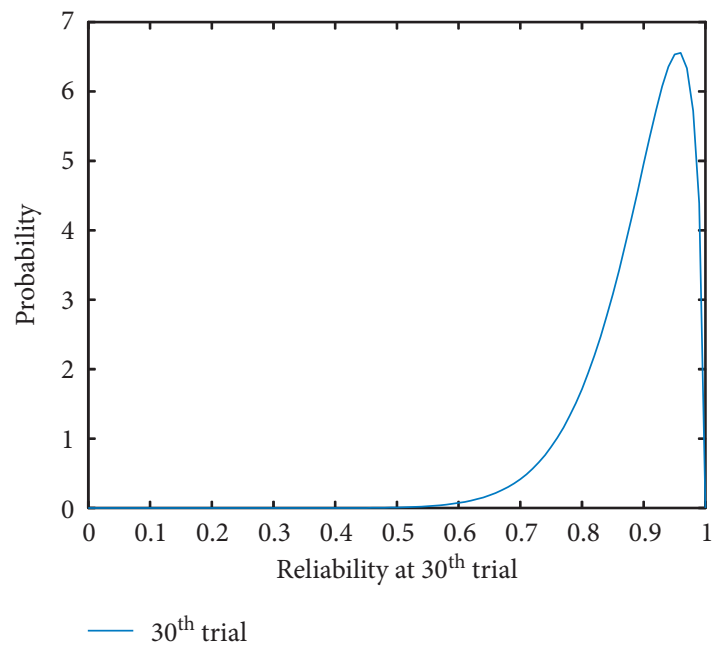

(f)

FIgURE 4: Reliability prediction at each trial. (a) Reliability estimated at the 5th trial. (b) Reliability estimated at the 10th trial. (c) Reliability estimated at the 15th trial. (d) Reliability estimated at the 20th trial. (e) Reliability estimated at the 25th trial. (f) Reliability estimated at the 30th trial. 
the 20th trial. This signifies that the failure rate of the products has been decreasing with the operational time. In the last row of Figure 4, the reliability of light-emitting diodes is increased noticeably, which is close to a value of 0.96 . This means that the light-emitting diodes achieve a very satisfactory level of quality through the testing process.

Figure 5 indicates the estimated reliability throughout the trial. It is observed that the estimated reliability decreases at the first five trials and reaches the lowest level at the 5th trial. During the early stage of the testing process, that is, from the 4th trial to the 10th trial, the reliability of lightemitting diodes is at relatively low level, and this situation has been changing since the 19th trial. The predicted reliability of light-emitting diodes eventually remains at a desirable level at the end of testing process.

This situation indicates that the new produced products are not stable regrading the operating quality at the beginning of service time and gradually become stable with a desirable level after some operational time. The result suggests that the industrial factories should spend this unstable time in production before the products come into service.

5.3. Reliability Control. Through the approach presented in Section 4, the 90\% confidence intervals for each trial are calculated (Figure 6). The upper limit of the prediction interval is 1 and the lower limit is plotted in Figure 6. We can see that the lower limit of the estimated reliability has the same evolution pattern as the mean reliability. In our reliability control approach, the control limit of lower limit of prediction interval is computed as 0.758 .

Figure 7 shows the reliability control process of the light-emitting diodes. In the figure, the control limit of reliability prediction interval is between 0.758 and 1 . The lower limit of the light-emitting diodes drops below the control limit within the 4th trial to the 6th trial, which indicates that a corresponding reliability control is required during the industrial production. After the 7th trial, the lower limit of $90 \%$ interval always remains above the control limit.

Through the above investigations, we have the two following conclusions: (1) The reliability of light-emitting diodes at early stage of production is not stable and will be stable with satisfactory level after some operational time, which signifies that the quality department should avoid this stage before the products come into service. (2) The reliability control method can maintain the distribution of lightemitting diodes reliability above a certain level, which helps to improve the production quality of the light-emitting diodes.

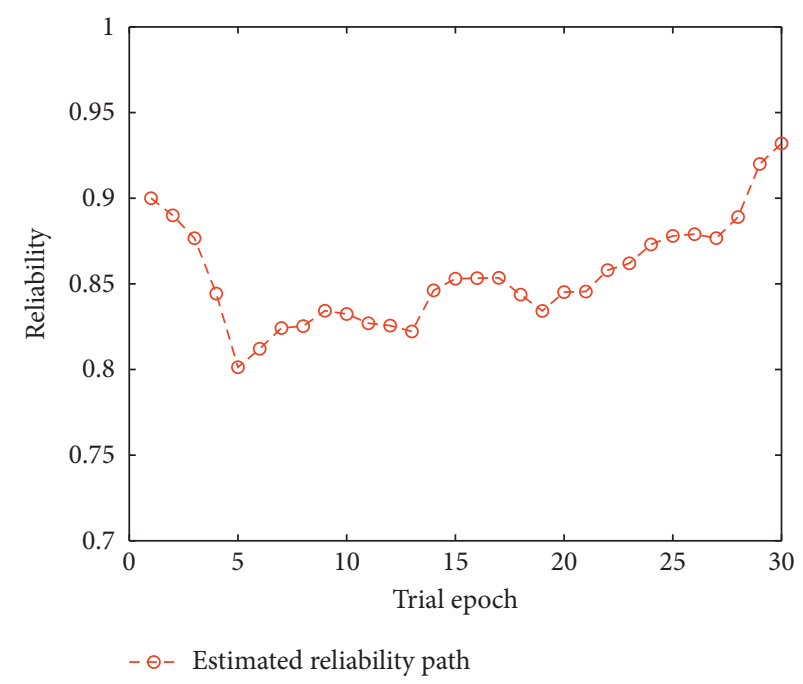

FIGURE 5: Estimated reliability path throughout the trial.

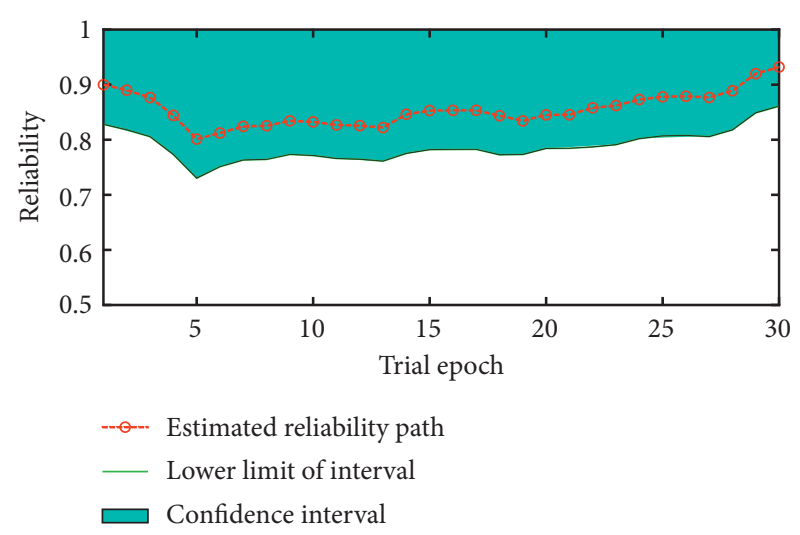

Figure 6: Estimated reliability interval for each trial.

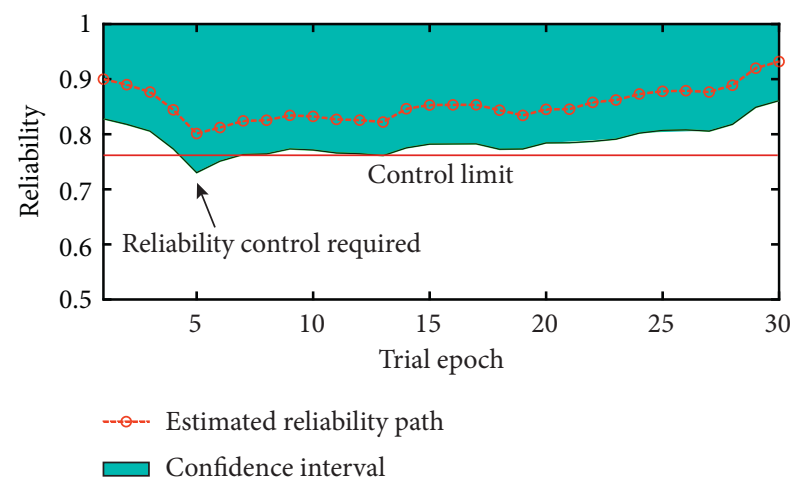

FIGURE 7: Illustration of reliability control process. 


\section{Conclusion}

This paper proposes a reliability estimation and control approach for deteriorating products under production process. The new manufactured products experience a series of failure testings at different operational times before real usage. To make full use of failure testing data, a Bayesian procedure is presented to estimate the reliability distribution of the industrial products. A point estimate is derived to represent the mean reliability of the products. The estimated reliability distribution and corresponding mean value are updated with new available testing data. Afterwards, a reliability control method is proposed to monitor the $90 \%$ prediction interval. When the lower limit of the $90 \%$ prediction interval drops below a control limit, a corresponding quality-assurance action is required. The control limit policy maintains the reliability of industrial products at a satisfactory level. Finally, a case of light-emitting diodes under failure trial at production process is illustrated for validating the proposed approach.

There are two interesting directions for future research works. The first direction is to consider the heterogeneity of the industrial products in current reliability estimation model, which would be valuable for real applications. Another interesting direction of our work is to consider the missing data existing among the failure trials which commonly occur in some factories and would be a useful and appealing topic for future study.

\section{Data Availability}

The data that support the findings of the research are available upon request from the corresponding author.

\section{Conflicts of Interest}

The authors declare that they have no conflicts of interest.

\section{Acknowledgments}

This research was supported by the National Natural Science Foundation of China (NSFC) under Grant no. 62033001.

\section{References}

[1] W. Booyse, D. N. Wilke, and S. Heyns, "Deep digital twins for detection, diagnostics and prognostics," Mechanical Systems and Signal Processing, vol. 140, Article ID 106612, 2020.

[2] E. Zio, "Reliability engineering: old problems and new challenges," Reliability Engineering \& System Safety, vol. 94, no. 2, pp. 125-141, 2009.

[3] S. Petchrompo and A. K. Parlikad, "A review of asset management literature on multi-asset systems," Reliability Engineering \& System Safety, vol. 181, pp. 181-201, 2019.

[4] Y. Chen, Z. Wang, Q. Ma, and K. Liang, "Reliability evaluation and failure behavior modeling of IMS considering functional and physical isolation effects," IEEE/ASME Transactions on Mechatronics, vol. 24, no. 6, pp. 2441-2452, 2019.

[5] E. Chiodo and D. Lauria, "Some basic properties of the failure rate of redundant reliability systems in industrial electronics applications," IEEE Transactions on Industrial Electronics, vol. 62 , no. 8, pp. 5055-5062, 2015.

[6] J. Z. Sikorska, M. Hodkiewicz, and L. Ma, "Prognostic modelling options for remaining useful life estimation by industry," Mechanical Systems and Signal Processing, vol. 25, no. 5, pp. 1803-1836, 2011.

[7] T. Aven, "Bayesian analysis: critical issues related to its scope and boundaries in a risk context," Reliability Engineering \& System Safety, vol. 204, Article ID 107209, 2020.

[8] B. Sun, X. Jiang, K. C. Yung, J. Fan, and M. G. Pecht, "A review of prognostic techniques for high-power white LEDs," IEEE Transactions on Power Electronics, vol. 32, no. 8, pp. 63386362, 2016.

[9] S. Alaswad and Y. Xiang, "A review on condition-based maintenance optimization models for stochastically deteriorating system," Reliability Engineering \& System Safety, vol. 157, pp. 54-63, 2017.

[10] C. Duan and C. Deng, "Prognostics of health measures for machines with aging and dynamic cumulative damage," IEEE/ ASME Transactions on Mechatronics, vol. 25, no. 5, pp. 2264-2275, 2020.

[11] D. R. Insua, F. Ruggeri, R. Soyer, and S. Wilson, "Advances in Bayesian decision making in reliability," European Journal of Operational Research, vol. 282, no. 1, pp. 1-18, 2020.

[12] Y. Lei, N. Li, L. Guo, N. Li, T. Yan, and J. Lin, "Machinery health prognostics: a systematic review from data acquisition to RUL prediction," Mechanical Systems and Signal Processing, vol. 104, pp. 799-834, 2018.

[13] J. G. Torres-Toledano and L. E. Sucar, "Bayesian networks for reliability analysis of complex systems," in Proceedings of the Ibero-American Conference on Artificial Intelligence, pp. 195-206, Springer, Lisbon, Portugal, October 1998.

[14] M. Asadi, N. Ebrahimi, and E. S. Soofi, "Optimal hazard models based on partial information," European Journal of Operational Research, vol. 270, no. 2, pp. 723-733, 2018.

[15] A. Heng, S. Zhang, A. C. C. Tan, and J. Mathew, "Rotating machinery prognostics: state of the art, challenges and opportunities," Mechanical Systems and Signal Processing, vol. 23, no. 3, pp. 724-739, 2009.

[16] M. C. Keizer, S. D. Flapper, and R. H. Teunter, "Condition-based maintenance policies for systems with multiple dependent components: a review," European Journal of Operational Research, vol. 261, no. 2, pp. 405-420, 2017.

[17] S. Song, D. W. Coit, Q. Feng, and H. Peng, "Reliability analysis for multi-component systems subject to multiple dependent competing failure processes," IEEE Transactions on Reliability, vol. 63, no. 1, pp. 331-345, 2014.

[18] W. Yao, X. Chen, Y. Huang, and M. Van Tooren, “An enhanced unified uncertainty analysis approach based on first order reliability method with single-level optimization," Reliability Engineering \& System Safety, vol. 116, pp. 28-37, 2013.

[19] P. V. Z. Cole, "A Bayesian reliability assessment of complex systems for binomial sampling," IEEE Transactions on Reliability, vol. R-24, no. 2, pp. 114-117, 1975.

[20] D. V. Mastran and N. D. Singpurwalla, "A Bayesian estimation of the reliability of coherent structures," Operations Research, vol. 26, no. 4, pp. 663-672, 1978.

[21] K. K. Sharma and R. K. Bhutani, "Bayesian reliability analysis of a parallel system," Reliability Engineering \& System Safety, vol. 37, no. 3, pp. 227-230, 1992.

[22] H. Bao, Y. Guo, H. Zhang, C. Peng, and J. Lu, "Bayesian analysis method on processing reliability data of high flux engineering test reactor," Reliability Engineering \& System Safety, vol. 199, Article ID 106912, 2020. 
[23] P. Gardner, C. Lord, and R. J. Barthorpe, "Bayesian history matching for structural dynamics applications," Mechanical Systems and Signal Processing, vol. 143, Article ID 106828, 2020.

[24] W. Peng, Y.-F. Li, J. Mi, L. Yu, and H.-Z. Huang, "Reliability of complex systems under dynamic conditions: a Bayesian multivariate degradation perspective," Reliability Engineering \& System Safety, vol. 153, pp. 75-87, 2016.

[25] S. Mahadevan, R. Zhang, and N. Smith, "Bayesian networks for system reliability reassessment," Structural Safety, vol. 23, no. 3, pp. 31-51, 2001.

[26] H. Langseth and L. Portinale, "Bayesian networks in reliability," Reliability Engineering \& System Safety, vol. 92, no. 1, pp. 92-108, 2007.

[27] H. Boudali and J. B. Dugan, "A discrete-time Bayesian network reliability modeling and analysis framework," Reliability Engineering \& System Safety, vol. 87, no. 3, pp. 337-349, 2005.

[28] D. Lee and R. Pan, "A nonparametric Bayesian network approach to assessing system reliability at early design stages," Reliability Engineering \& System Safety, vol. 171, pp. 57-66, 2018.

[29] T.-T. Liu, R. Liu, and G.-J. Duan, "A principle-empirical model based on Bayesian network for quality improvement in mechanical products development," Computers \& Industrial Engineering, vol. 149, Article ID 106807, 2020.

[30] B. Cai, X. Kong, Y. Liu et al., "Application of Bayesian networks in reliability evaluation," IEEE Transactions on Industrial Informatics, vol. 15, no. 4, pp. 2146-2157, 2019.

[31] S. Rebello, H. Yu, and L. Ma, "An integrated approach for system functional reliability assessment using dynamic Bayesian network and Hidden Markov model," Reliability Engineering \& System Safety, vol. 180, pp. 124-135, 2018.

[32] J. Neyman, Lectures and Conferences on Mathematical Statistics and Probability, Graduate School, US Department of Agriculture, Washington, DC, USA, 1952.

[33] C. M. Thompson, E. S. Pearson, L. J. Comrie, and H. O. Hartley, Tables of Percentage Points of the Incomplete Beta-Function, Biometrika, Cattolica, Italy, 1941.

[34] H. C. Tijms, A First Course in Stochastic Models, John Wiley and Sons, Hoboken, NJ, USA, 2003. 